\title{
RPB5-Mediating Protein Suppresses Hepatitis B Virus (HBV) Transcription and Replication byCounteracting theTranscriptional Activation of Hepatitis B virus X Protein in HBV Replication Mouse Model
}

\author{
Qiaoling Zhou $^{1,2}$; Feijun Huang ${ }^{3}$; Lanlan Chen $^{1,2}$; Enqiang Chen ${ }^{1,2}$; Lang Bai ${ }^{1,2}$; Xing Cheng ${ }^{1,2}$; \\ Min $\mathrm{He}^{1,2}$; Hong Tang ${ }^{1,2,}$ \\ ${ }^{1}$ Center of Infectious Diseases, West China Hospital, Sichuan University, Chengdu, Sichuan, Republic of China \\ ${ }_{2}^{2}$ Division of Infectious Diseases, State Key Laboratory of Biotherapy, Sichuan University, Chengdu, Sichuan, Republic of China \\ ${ }^{3}$ Department of Forensic Pathology, Medical School of Basic and Forensic Sciences, Sichuan University, Chengdu, Republic of China \\ ${ }^{*}$ Corresponding author: Hong Tang, Center of Infectious Diseases, West China Hospital, Sichuan University, Chengdu, Sichuan, Republic of China. Tel:+86-2885422650, Fax:+86-2885423052, \\ E-mail: htang6198@hotmail.com \\ Received: July 11, 2014; Revised: November 11, 2014; Accepted: December 19, 2014
}

\begin{abstract}
Background: RPB5-Mediating protein (RMP) is associated with the RNA polymerase II subunit RPB5. This protein functionally counteracts the transcriptional activation of Hepatitis B Virus X protein ( $\mathrm{HBx}$ ) by competitively binding to the RPB5; however, the effects of RMP on Hepatitis B virus (HBV) transcription and replication remain unknown.

Objectives: The purpose of this study was to investigate the effect of RMP on viral transcription and replication in vivo by using the hydrodynamic-based HBV replication mouse model.

Materials and Methods: Male balb/c mice were transfected with wild type (1.2 wt) or the HBx minus HBV plasmids (1.2x (-)) with or without HBx and RMP, to establish an HBV replication mouse model by hydrodynamic injection through the tail vein. The HBV RNA and HBV DNA replication intermediates (RI) were analyzed in the liver.

Results: RPB5-Mediating protein could inhibit HBV transcription and replication in groups transfected with the $1.2 \mathrm{wt}$ and HBx. The inhibitory effect disappeared in the $1.2 \mathrm{x}(-)$ groups, yet it reappeared in the groups co-transfected with $1.2 \mathrm{x}(-)$ and HBx. An inhibitory effect was indicated at a low dose of RMP ( $0.3 \mathrm{ug}, 0.5 \mathrm{ug}$ and $0.7 \mathrm{ug})$ compared to the control group and groups that had received high doses of RMP.

Conclusions: Our study demonstrated that a low dose of RMP could inhibit HBV transcription and replication, which is dependent on the appearance of $\mathrm{HBx}$ in vivo.
\end{abstract}

Keywords: URI 1 Protein; Orthohepadnavirus; Hepatitis B Virus X Protein; Virus Replication

\section{Background}

Hepatitis B virus $\mathrm{X}$ protein (HBx) is a 154-amino acid protein and has been reported as a multifunctional protein that exhibits effects on gene transcription, signaling pathways, genotoxic stress responses, protein degradation, cell-cycle control, cell proliferation and apoptosis (1-3). The role of $\mathrm{HBx}$ in the viral life cycle was addressed by several experimental systems. It has been shown that HBx is needed for successful establishment of Woodchuck Hepatitis virus (WHV) infection in vivo $(4,5)$. However, its essential role in Hepatitis $B$ virus (HBV) life cycle is controversial. Furthermore, HBX-deficiency had little effect in HBV replication in human hepatoma Huh7 cells $(6,7)$, yet replication was impaired in hepG 2 cells $(8,9)$. These may suggest that HBx has a critical role for establishment of virus infection yet not for establishment of viral replication (10). With the establishment of a plasmid-based HBV replication assay, it is now known that HBx enhances HBV transcription and replication in vitro and in vivo, although the mechanism by which HBx facilitates HBV replication remains unclear $(8,11-14)$.

Since HBx cannot bind DNA directly, protein-protein interaction is critical for $\mathrm{HBx}$ transactivation. Hepatitis $B$ virus $X$ protein interacts with several cellular proteins, and its role in virus transcription and replication may be mediated through these interactions $(3,15)$. The direct interactions of HBx with general Transcription Factor IIB (TFIIB) and the RNA Polymerase II subunit 5 (RPB5) are critical for the coactivation ability of HBx, which augments HBV replication positively and modulates gene expression of cells $(16,17)$.

The exposed domain of RPB5 is involved in interactions with transcriptional regulators including HBx (18), TFIIB (19), general transcription factor IIF (TFIIF) (20) and RPB5Mediating Protein (RMP)/Unconventional RPB5-interacting protein (URI) (21). RPB5-Mediating protein is a RPB5 associated protein with 508 amino acids. The RMP gene is conserved among mammals and ubiquitously expressed in

Copyright (c) 2015, Ahvaz Jundishapur University of Medical Sciences. This is an open-access article distributed under the terms of the Creative Commons Attribution-NonCommercial 4.0 International License (http://creativecommons.org/licenses/by-nc/4.0/) which permits copy and redistribute the material just in noncommercial usages, provided the original work is properly cited. 
various tissues (21). Multiple RMP variants or homologues have been reported in humans and other species. Unconventional RPB5-interacting protein, an alternative form of RMP, was shown to participate in a nutrients-related signaling pathway that is required for gene expression (22). The $\mathrm{RMP} / \mathrm{URI}$ is a component of the multiprotein PAF1 complex that is involved in histone methylation, gene expression and cell cycle regulation (23). As a major regulator of transcription and cell cycle, RMP is associated with PRB5 and other transcriptional regulators such as HBx and TFIIB. In previous studies it has been shown that RMP can not directly bind to transcriptional regulators such as $\mathrm{HBx}$ and TFIIB, yet the RMP binding region of RPB5 (aa 1 to 160), which is more than two-thirds of RPB5, overlaps with the TFIIB and HBx binding sites (aa 1 to 46 and 47 to 120 , respectively) and RMP may interfere with the association of TFIIB, HBx and RPB5 (21). In a previous research it was shown that on the one hand the RMP can counteract transactivation through $\mathrm{HBX}$,on the other hand the overexpression of HBx could reduce the inhibitory effect of PMP on transcription (21). However, the effect of RMP on HBV transcription and replication is unknown.

\section{Objectives}

The current study aimed to investigate the effect of RMP on in vivo viral life cycle by using a hydrodynamic-based HBV replication mouse model.

\section{Materials and Methods}

\subsection{Ethics Statement}

This study obtained ethics approval from the laboratory of animal ethics committee of Sichuan university.

\subsection{Plasmids}

The plasmid payw1.2 (1.2 wt) contains 1.2 copies of the wild-type HBV (subtype ayw) genome and expresses HBV pre-genomic 3.5 Kb RNA under the control of the endogenous promoter of HBV (11). The plasmid payw * $7(1.2 \times(-))$ is a HBx-minus mutant vector, which contains an ochre termination signal (CAA to UAA) after codon 7 (at codon 8 ) in the HBx ORF, and has been derived from payw1.2 by site-directed mutagenesis (11). The pNKF-HBx vector expresses full-length HBx (aa 1 to 154) (16). The plasmid pSG5UTL-RMP expresses full-length RMP (aa 1to 508) (21).

\subsection{Establishment of Hepatitis B Virus Replication Mouse Model by Hydrodynamic Injection}

Male BALB/C mice at specific pathogen free (SPF), weighing 18 to $20 \mathrm{~g}$, were provided by the Huaxi laboratory animal center of Sichuan university. Mice were injected via the tail vain with plasmids in phosphate-buffered saline (PBS) to a volume equivalent to $8 \%$ of total body weight of each animal during five to eight seconds $(24,25)$. All mice received humane care under the Institutional Review Board in accordance with the Animal Protection Art of Sichuan University. There were three groups in this study 1) $1.2 \mathrm{wt}+\mathrm{HBx}, 2) 1.2 \mathrm{x}(-), 3) 1.2 \mathrm{x}(-)+\mathrm{HBx}$; each group was injected with $10 \mu \mathrm{g}$ of payw1.2 $(1.2 \mathrm{wt})$ or payw $\times 7(1.2 \mathrm{x}$ $(-))$ with or without $1 \mu \mathrm{g}$ of pNKF-HBx and the same dose of pSG5UTL-RMP. The mice were sacrificed 72 hours after injection. Their liver tissue was frozen in liquid nitrogen and stored at $-70^{\circ} \mathrm{C}$ prior to analysis for HBV RNA and HBV DNA replication intermediates (RI). Frozen liver tissue was mechanically pulverized in liquid nitrogen and used for preparation of total cellular RNA and HBV DNA RI.

\subsection{Analysis of Hepatitis B Virus RNA by Northern Blotting}

Total RNA was isolated using TRIZOL (Invitrogen, USA) reagent according to the manufacturer's instructions. Next, 30 ug of total RNA was separated on 1\% formaldehyde gel and transferred to a positively charged nylon membrane (Amersham, USA). The membranes were probed with digoxigenin-labeled full-length HBV DNA to detect HBV sequence; the mouse glyceraldehydes 3-Phosphate dehydrogenase (GAPDH) cDNA was used as an internal control and membrane hybridization was detected using Digoxin (DIG) high prime DNA labeling and detection starter kit II (Roche Applied Science, USA) and X-ray film.

\subsection{Analysis of HBVDNA Replication Intermediates by Southern Blotting}

Frozen liver tissues were mechanically pulverized in liquid nitrogen and HBV replication intermediates were isolated form $120 \mu \mathrm{g}$ of liver tissue powder, as described previously (11). These HBV DNA replication intermediates (HBV DNA RI) were diluted in $30 \mu$ lof Tris-Ethylene diamine tetraacetic acid (TE) buffer. The HBV replication intermediates were separated on $1 \%$ agarose gels and transferred to a positively charged nylon membrane. The membranes were probed with digoxigenin-labeled full-length HBV DNA to detect HBV sequence, and membrane hybridization was detected using DIG high prime DNA labeling and detection starter kit II (Roche Applied Science, USA) and X-ray film.

\subsection{Quantitation}

Northern blotting and southern blotting were exposed on Kodak BioMax MS film and quantitated by the quantity one system.

\section{Results}

\subsection{RPB5-Mediating Protein Inhibits the Transcrip- tion and Replication of Hepatitis B Virus in Groups Co-transfected With 1.2 wt and $H B x$}

To investigate the effect of RMP on the replication of $\mathrm{HBV}$ in vivo, we divided the mice into five groups; each 
group received plasmids of payw1.2 and HBx plus different doses of RMP (0, 0.3, 0.5, 0.7 and 1.0 ug, respectively). As shown in Figure $1 \mathrm{~A}$ and $\mathrm{C}$, compared to the control group, the groups that received a low dose of RMP had lower level of $3.5 \mathrm{~Kb}$ RNA, which were decreased to $32.5 \%$, $34.9 \%$ and $81.5 \%$ in the $0.3 \mathrm{ug}, 0.5 \mathrm{ug}$ and $0.7 \mathrm{ug}$ RMP groups, respectively. The level of HBV RNA had no difference between the control group and the high dose group (decreased to $89.4 \%$ ). The level of HBV DNA RI in these groups is shown in Figure $1 \mathrm{~B}$ and $\mathrm{D}$; compared to the control group, the groups that received RMP had decreased levels of HBV DNA RI by $43.9 \%, 69.2 \%, 59.7 \%$ and $96.2 \%$ with a dose of $0.3 \mathrm{ug}, 0.5 \mathrm{ug}, 0.7 \mathrm{ug}$ and $1.0 \mathrm{ug}$ of RMP, respectively.

These results suggested that a low dose of RMP could in- hibit the transcription and replication of HBV; however as the dose increased the effect disappeared.

\subsection{The Inhibitory Effect of RPB5-Mediating Pro-} tein Disappeared When The Mice were Transfected With HBx Minus Plasmids

To investigate whether the RMP directly influences HBV transcription and replication, we injected the HBx minus payw1.2 $\times 7$ plasmid and plasmid vector $\mathrm{pNKF}$ plus different doses of RMP. As shown in Figure 2, compared to the control group, the level of $3.5 \mathrm{~Kb}$ RNA was $100.0 \%, 106.8 \%$, 96.7\% and 99.6\%, while the level of HBV DNA RI was $108.7 \%$, $106.8 \%, 87.7 \%$ and $97.5 \%$ in the groups with $0.3 \mathrm{ug}, 0.5 \mathrm{ug}$, $0.7 \mathrm{ug}$ and $1.0 \mathrm{ug}$ of RMP, respectively.

Figure 1. RPB5-Mediating Protein Inhibition of the Transcription and Replication of Hepatitis B Virus in Groups Co-transfected With 1.2 wt and HBx

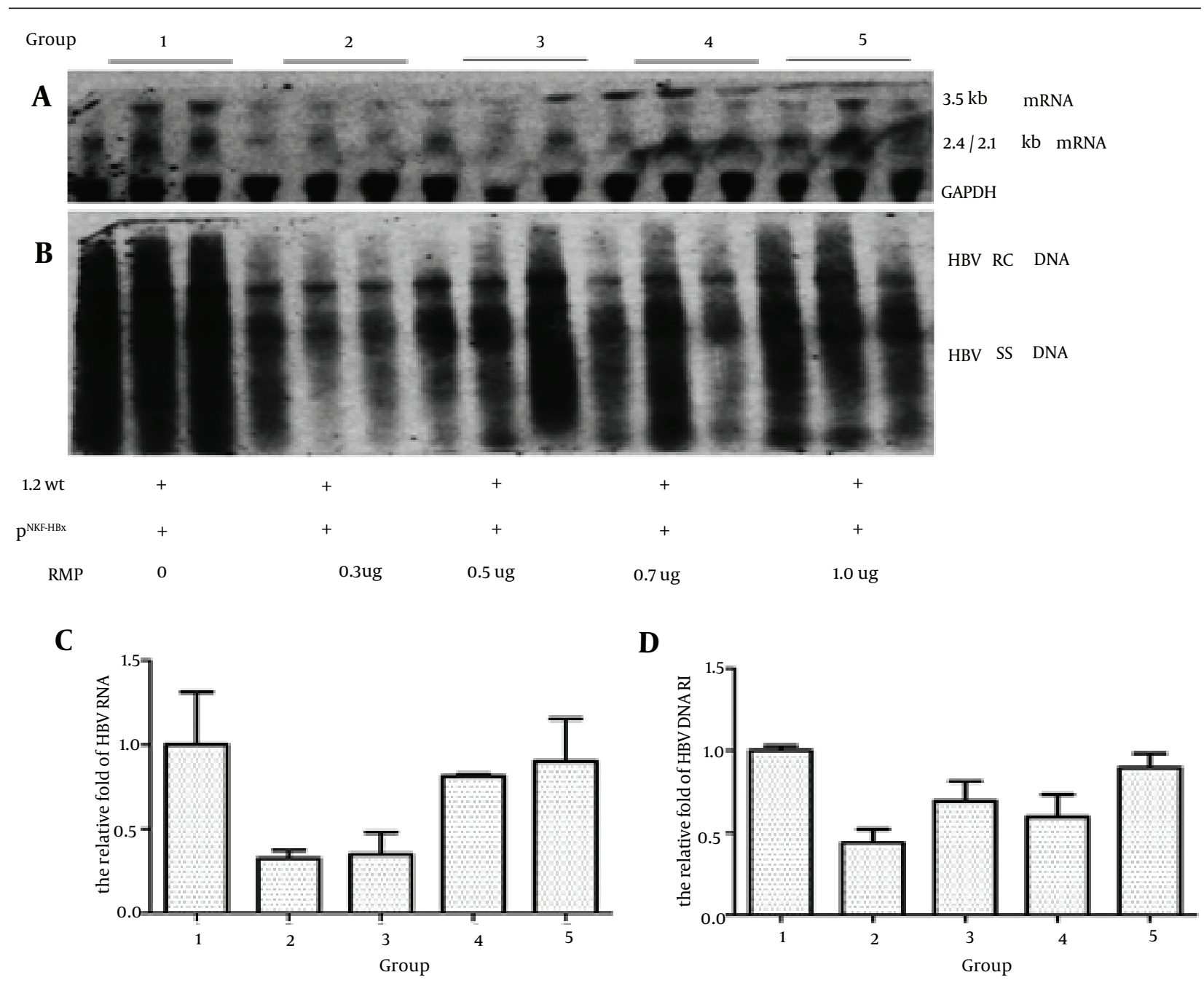

Mice were injected with $10 \mathrm{ug}$ of payw1.2 (1.2 wt) and $1 \mathrm{ug}$ of pNKF-HBx plus different amounts of pSG5UTL-RMP (0, 0.3 ug, 0.5 ug, 0.7 ug and 1.0 ug, respectively). The next steps were in the following order, A, HBV RNA of mice liver was detected by northern blotting and the GAPDH RNA was used as an internal control for RNA loading per lane; B, HBV DNA RI was detected by southern blotting; C, Quantitative analysis of the 3.5 kb HBV RNA; D, Quantitative analysis of HBV DNA RI. 
The levels of 3.5 Kb RNA and HBV DNA RI had no significant differences among these groups. These results suggested that in absence of HBx, RMP had no inhibitory effect on HBV transcription and replication. In addition, as indicated by Figure 1, compared to the control group the virus could still replicate in the mice liver when transfected with HBx minus plasmids, yet with a very low level of HBV transcription and replication.

\subsection{The Inhibitory Effect After Co-transfection with $\mathrm{HBX}$ Minus Plasmids and $\mathrm{HBX}$}

To investigate whether HBx played a critical role in the inhibition of RMP during HBV transcription and replication, we injected the HBx minus payw1. $2 \times 7$ plasmids with pNKF-HBx plus different levels of RMP. As shown in Figure 3, firstly, compared to the control group, the level of $3.5 \mathrm{~Kb}$ RNA was $52.0 \%, 35.8 \%, 56.8 \%$ and $101.4 \%$ while the level of HBV DNA RI was $34.2 \%, 58.4 \%, 48.7 \%$ and $98.8 \%$ in the presence of $0.3 \mathrm{ug}, 0.5 \mathrm{ug}, 0.7 \mathrm{ug}$ and $1.0 \mathrm{ug}$ of RMP, respectively. In the low dose RMP groups, the level of HBV transcription and replication intermediate was partly inhibited compared to the control and high dose RMP groups. Secondly, as indicated by Figures 1 and 2, compared to control group, the HBx restored the capacity of HBx minus HBV transcription and replication in the wild type.

Figure 2. The Inhibitory Effect After Transfection With HBx Minus Plasmids

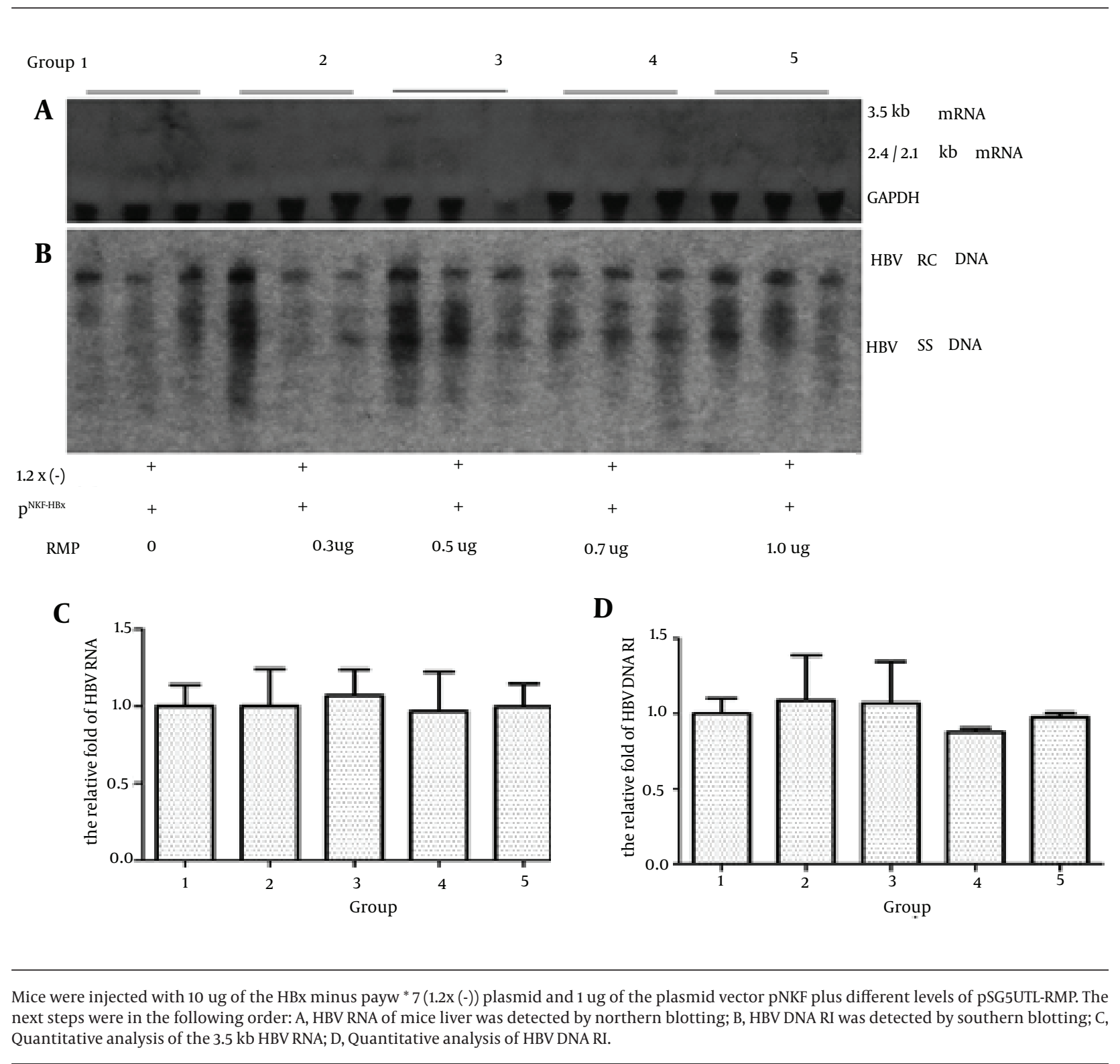



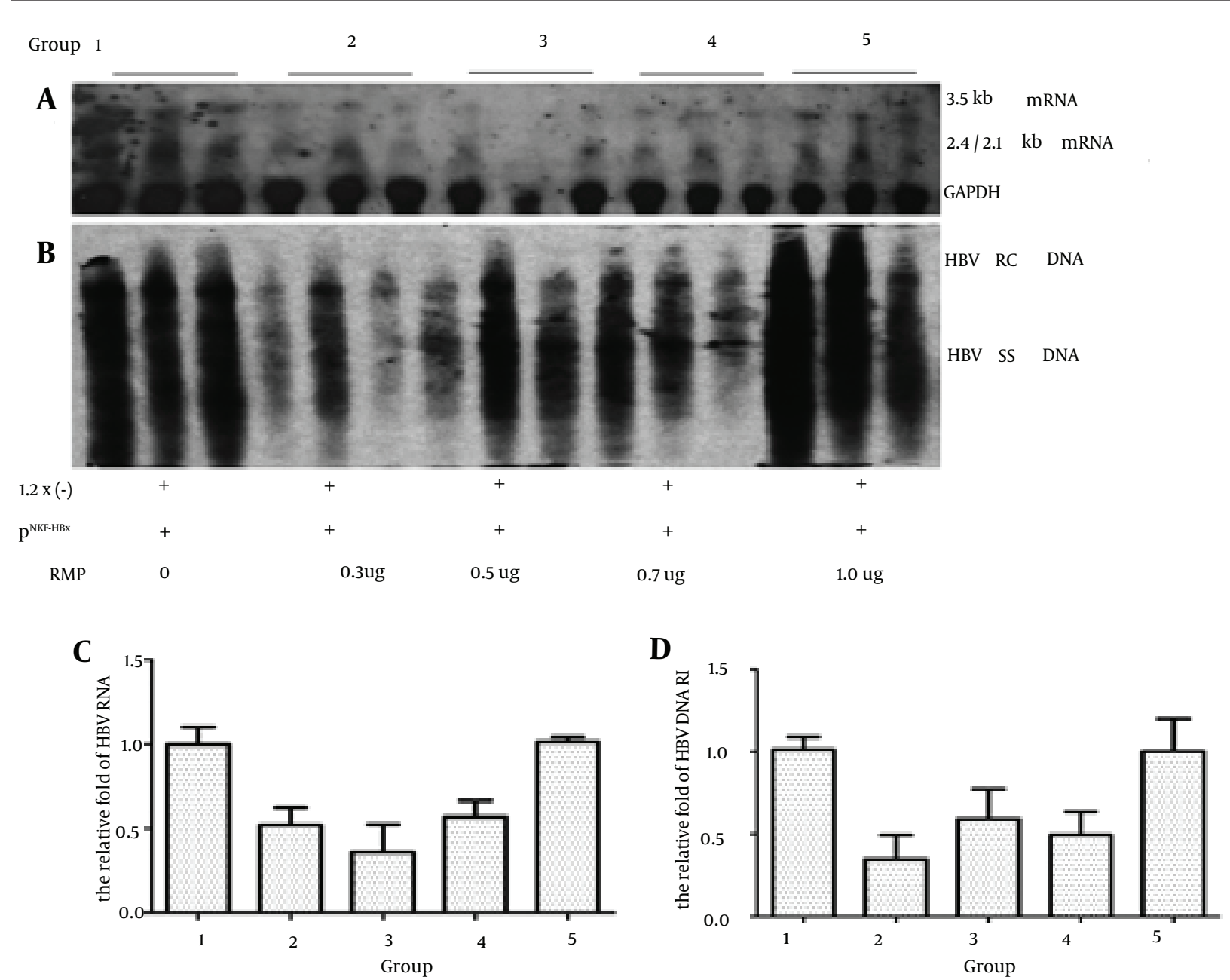

Mice were injected 10 ug of the HBx minus payw ${ }^{*} 7(1.2 x(-))$ plasmids with 1 ug of pNKF-HBx plus different levels of pSG5UTL-RMP. The next steps were in the following order:A, HBV RNA of mice liver was detected by northern blotting; B, HBV DNA RI was detected by southern blotting; C, Quantitative analysis of the 3.5kb HBV RNA; D, Quantitative analysis of HBV DNA RI.

\section{Discussion}

Hepatitis B virus X protein is a trans-activator that regulates a wide range of cellular and viral genes transcribed by RNA polymerase II and III. As HBx is unable to bind to double stranded DNA, it may directly modulate transcription through interaction with a variety of transactivators or factors involved in transcription (26). Previous studies have found that HBx specifically binds to RPB5, a common subunit shared by eukaryotic nuclear RNA polymerases I, II and III, and transcription factor TFIIB, and that the trimeric interactions may be involved in HBx transactivation (17). RPB5-Mediating protein is a RPB5 associated protein and specifically binds to RPB5. As RMP and the other transcriptional regulators like $\mathrm{HBx}$ and TFIB cannot bind to each other, RMP regulates the transactivation of these transcriptional regulators by competing for binding to RPB5 (21). In a previous study, HepG2 cells were transiently co- transfected with RMP and HBx expression plasmids together with the pHEC $\times 2$ CAT, an HBx-responsive chloramphenical acetyltransferease (CAT) reporter. In this study RMP could counteract transactivation by HBx, and the overexpression of $\mathrm{HBx}$ reduced the inhibitory effect of RMP on transcription and in the absence of HBx the inhibitory effect of RMP could not work. (21). However, the level of CAT activity only reflected the transactivation of HBx yet not the level of HBV transcription and replication. In our study, RMP could inhibit HBV transcription and replication and this inhibitory effect depended on the appearance of HBx. The negative effects of RMP on HBV transcription and replication disappeared with the absence of HBx. When the groups were transfected with the HBx-minus plasmid (payw $\times 7$ ) (Figure 2), RMP had no inhibitory effect on HBV transcription and replica- 
tion in vivo; while inhibitory effects were seen in groups co-transfected with the HBx plasmid (Figures 1 and 3). However, there were no obvious inhibitory effects in the groups transfected with the wild-type plasmid plus different doses of RMP (data not show). The dose of HBx may have played an important role in the differences of results between the two groups transfected with the wild-type plasmid with or without HBx. The dose of HBx was nearly undetectable in the groups that were only transfected with the wild type plasmids in vitro and in vivo, being much higher in the group transfected with the exogenous HBx plasmid (8). This suggested that RMP inhibited HBV transcription and replication, which is dependent on the appearance of $\mathrm{HBx}$, and the dose of $\mathrm{HBx}$ may have affected the inhibitory mechanisms of RMP on HBV transcription and replication.

An inhibitory effect was indicated in groups that received a low dose of RMP. However, previous studies reported that RMP negative affected a wide variety of activated transcriptions in a dose-depended manner in vitro. In the HepG2 cells co-transfected with the RMP and HBx or Gal-VP16, RMP counteracted the transcription activation of HBx and Gal-VP16, in a dose-depended manner (21). However, in our replication mouse model, the inhibitory effect of RMP on HBV transcription and replication was only observed at a low dose of RMP. The capacity of HBV transcription and replication was partially restored in the high dose groups compared to the low dose groups.

As the RMP and HBx cannot directly bind to each other, RMP counteracted HBx transcription activation by competitive binding to RPB5. Firstly, RMP is mainly localized in the cytoplasm, accompanied by weak and diffuse nuclear localization (27). However, HBx needs to localize the nucleus to enhance or restore the capacity of HBV replication (28, 29). The overexpression of RMP didn't mean an increase in nuclear localization. Thus, the spatial relationship between RMP, HBx and RPB5 may affect the inhibitory effect of RMP on the capacity of HBV replication and transcription. Secondly, it is possible that RMP requires signaling processes for its function and a functional partner for its function in addition to RPB5 binding. Similar to the RMP interacting protein, DMAP1, this protein facilitates nuclear localization of RMP and augments the corepressor activity of RMP (27). Thirdly, there are several studies that have reported that RMP plays an important role in cell cycle and pathways controlling apoptosis. In humans, RMP/URI is a component of the multi-protein PAF1 complex that is involved in histone methylation, gene expression and cell cycle regulation(23). Hepatitis B virus particles released from apoptotic hepatocytes were immature and non-enveloped and proved not to be infectious. Furthermore, HBV needs to prevent apoptosis of its host hepatocyte to ensure the release of infectious progeny and, thus virus spread in the liver (30). Future studies could test the expression of RMP in the nuclear and cytoplasm of the mice liver by western blot or Immunohistochemistry (IHC) after injection, and detect the life cycle of HepG2 cells by flow cytometry (FCM) after transfection with HBV and RMP plasmids. As RMP affects a wide variety of activated transcriptions and plays an important role in cell cycle and pathways, by controlling apoptosis we could determine its' role in hepatocellular carcinoma, in the future.

Previous researches suggested that HBx has a critical role for establishment of virus infection yet not for establishment of viral replication (10). However, in our study, the hydrodynamic animal model of HBV was a replication model and the replication of virus in the mice liver only lasts for a few days (24). Therefore, in our study, we detected HBV RNA and HBV DNA RI in the mice liver after injection with the HBx minus plasmid payw $\times 7$, and compared this group to mice injected with the wild-type or the payw $\times 7$ plus HBx; the level of HBV RNA and HBV DNA RI was observably lower in the payw $\times 7$ groups. These results suggested that HBx does not have an essential role in HBV replication, yet it can observably enhance the capacity of HBV transcription and replication. Despite residual replication in the absence of $\mathrm{HBx}$, the effect of HBx-deficiency was reproducible and could be complemented by the addition of a second plasmid encoding HBx; this was confirmed by previous studies $(8,28,31)$.

In conclusion, although further investigation is required, our study found that inhibition of low dose RMP on HBV transcription and replication depended on the appearance of HBx in vivo and suggested that RMP played important roles in the regulation of HBV life cycle. This function might facilitate RMP as a candidate target molecule in the treatment of HBV.

\section{Acknowledgements}

We are grateful to Seishi Murakami (department of molecular oncology, cancer research institute, Kanazawa university, Kanazawa 920-0934, Japan) for providing the plasmid of $1.2 \mathrm{wt}, 1.2 \mathrm{x}(-)$ and HBx, and RMP.

\section{Authors' Contributions}

Hong Tang and Feijun Huang designed the research and provided fundamental support. Qiaoling Zhou, Lanlan Chen, Enqiang Chen and Xing Cheng performed the research. Qiaoling Zhou, Lang Bai and Min He analyzed the data. Qiaoling Zhou wrote the paper.

\section{Financial Disclosure}

This project was funded by the national natural science foundation of China under grants No. 81071363, No. 30570064 and No. 2013CB911300. The funders had no role in the study design, data collection and analysis, decision to publish or preparation of the manuscript.

\section{Funding/Support}

This work was partially supported by the national natural science foundation of China (No. 81071363 and No. 30570064) and the national basic research program of China (No. 2013CB911300). 


\section{References}

1. Bouchard MJ, Schneider RJ. The enigmatic X gene of hepatitis B virus. J Virol. 2004;78(23):12725-34.

2. Tang H, Oishi N, Kaneko S, Murakami S. Molecular functions and biological roles of hepatitis B virus x protein. Cancer Sci. 2006;97(10):977-83.

3. Murakami S. Hepatitis B virus X protein: a multifunctional viral regulator. J Gastroenterol. 2001;36(10):651-60.

4. Chen HS, Kaneko S, Girones R, Anderson RW, Hornbuckle WE, Tennant BC, et al. The woodchuck hepatitis virus $\mathrm{X}$ gene is important for establishment of virus infection in woodchucks. J Virol. 1993;67(3):1218-26.

5. Zoulim F, Saputelli J, Seeger C. Woodchuck hepatitis virus X protein is required for viral infection in vivo. JVirol. 1994;68(3):2026-30.

6. Blum HE, Zhang ZS, Galun E, von Weizsacker F, Garner B, Liang TJ, et al. Hepatitis B virus X protein is not central to the viral life cycle in vitro. J Virol.1992;66(2):1223-7.

7. Gong DY, Chen EQ, Huang FJ, Leng XH, Cheng X, Tang H. Role and functional domain of hepatitis $\mathrm{B}$ virus $\mathrm{X}$ protein in regulating HBV transcription and replication in vitro and in vivo. Viruses. 2013;5(5):1261-71.

8. Keasler VV, Hodgson AJ, Madden CR, Slagle BL. Enhancement of hepatitis $\mathrm{B}$ virus replication by the regulatory $\mathrm{X}$ protein in vitro and in vivo. J Virol. 2007;81(6):2656-62.

9. Xu Z, Yen TS, Wu L, Madden CR, Tan W, Slagle BL, et al. Enhancement of hepatitis B virus replication by its X protein in transgenic mice. J Virol. 2002;76(5):2579-84.

10. Lucifora J, Arzberger S, Durantel D, Belloni L, Strubin M Levrero M, et al. Hepatitis B virus X protein is essential to initiate and maintain virus replication after infection. J Hepatol. 2011;55(5):996-1003.

11. Tang H, Delgermaa L, Huang F, Oishi N, Liu L, He F, et al. The transcriptional transactivation function of HBx protein is important for its augmentation role in hepatitis B virus replication.J Virol. 2005;79(9):5548-56.

12. Belloni L, Pollicino T, De Nicola F, Guerrieri F, Raffa G, Fanciulli M, et al. Nuclear HBx binds the HBV minichromosome and modifies the epigenetic regulation of cccDNA function. Proc Natl Acad Sci U S A. 2009;106(47):19975-9.

13. Tang KF, Xie J, Chen M, Liu Q, Zhou XY, Zeng W, et al. Knockdown of damage-specific DNA binding protein 1 (DDB1) enhances the HBx-siRNA-mediated inhibition of HBV replication. Biologicals. 2008;36(3):177-83.

14. Hao M, Zheng S, Ding H, Huang A. [Regulation of microRNA-122 on HBV replication by targeting HBx sequence]. Sheng Wu Yi Xue Gong Cheng Xue Za Zhi. 2011;28(4):784-9.

15. Murakami S. Hepatitis B virus X protein: structure, function and biology. Intervirology. 1999;42(2-3):81-99.

16. Lin Y, Nomura T, Cheong J, Dorjsuren D, Iida K, Murakami S. Hepatitis $B$ virus $X$ protein is a transcriptional modulator that communicates with transcription factor IIB and the RNA polymerase II subunit 5. J Biol Chem. 1997;272(11):7132-9.

17. Yang Y, Ma Y, Zhen L, Chen Y, Ma W, Murakami S. HBV X protein
(HBX) interacts with general transcription factor TFIIB both in vitro and in vivo. Chin Med Sci J.1999;14(3):152-7.

18. Cheong JH, Yi M, Lin Y, Murakami S. Human RPB5, a subunit shared by eukaryotic nuclear RNA polymerases, binds human hepatitis $\mathrm{B}$ virus $\mathrm{X}$ protein and may play a role in $\mathrm{X}$ transactivation. EMBO J.1995;14(1):143-50.

19. Yang Y, Zheng L, Chen Y. [Study of HBV X protein and RMP, an RPB5 mediate protein competitively interacting with general transcription factor TF2B]. Zhonghua Gan Zang Bing Za Zhi. 2000;8(1):15-7.

20. Wei W, Gu JX, Zhu CQ, Sun FY, Dorjsuren D, Lin Y, et al. Interaction with general transcription factor IIF (TFIIF) is required for the suppression of activated transcription by RPB5-mediating protein (RMP). Cell Res. 2003;13(2):111-20.

21. Dorjsuren D, Lin Y, Wei W, Yamashita T, Nomura T, Hayashi N, et al. RMP, a novel RNA polymerase II subunit 5-interacting protein, counteracts transactivation by hepatitis B virus X protein. Mol Cell Biol. 1998;18(12):7546-55.

22. Gstaiger M, Luke B, Hess D, Oakeley EJ, Wirbelauer C, Blondel M, et al. Control of nutrient-sensitive transcription programs by the unconventional prefoldin URI. Science. 2003;302(5648):1208-12.

23. Yart A, Gstaiger M, Wirbelauer C, Pecnik M, Anastasiou D, Hess D, et al. The HRPT2 tumor suppressor gene product parafibromin associates with human PAF1 and RNA polymerase II. Mol Cell Biol. 2005;25(12):5052-60.

24. Liu FJ, Liu L, He F, Wang S, Zhou TY, Liu C, et al. Establishment and primary application of a mouse model with hepatitis B virus replication. World J Gastroenterol. 2007;13(40):5324-30.

25. Gao Z, Liu FJ, Liu L, Zhou TY, Lei J, Xu L, et al. Application of hepatitis B virus replication mouse model. World J Gastroenterol. 2010;16(16):1979-85.

26. Yen TS. Hepadnaviral X Protein:Review of Recent Progress. J Biomed Sci.1996;3(1):20-30.

27. Delgermaa L, Hayashi N, Dorjsuren D, Nomura T, Thuy le TT, Murakami S. Subcellular localization of RPB5-mediating protein and its putative functional partner. Mol Cell Biol. 2004;24(19):8556-66.

28. Keasler VV, Hodgson AJ, Madden CR, Slagle BL. Hepatitis B virus HBx protein localized to the nucleus restores HBx-deficient virus replication in HepG 2 cells and in vivo in hydrodynamically-injected mice. Virology. 2009;390(1):122-9.

29. Lee JH, Rho HM. Nuclear factor of activated T cells (NFAT1-C) represses the enhancer II and pregenomic promoter (EnII/CP) of hepatitis B virus (HBV) through its responsive site GGAGA and nullifies the HBx-driven transcriptional activation. IUBMB Life. 2001;51(4):255-61.

30. Arzberger S, Hosel M, Protzer U. Apoptosis of hepatitis B virusinfected hepatocytes prevents release of infectious virus. J Virol. 2010;84(22):11994-2001

31. Hodgson AJ, Hyser JM, Keasler VV, Cang Y, Slagle BL. Hepatitis $B$ virus regulatory $\mathrm{HBx}$ protein binding to DDB1 is required but is not sufficient for maximal HBV replication. Virology. 2012;426(1):73-82. 